\title{
Analytical characteristics and comparative evaluation of Aptima HIV-1 Quant Dx assay with Ampliprep/COBAS TaqMan HIV-1 test v2.0
}

Angelos. Hatzakis ${ }^{1,2,4^{*}}$, Helen Papachristou', Sangeetha J. Nair ${ }^{3}$, Jacqueline Fortunko ${ }^{3}$, Tracy Foote ${ }^{3}$, HeeCheol Kim³ Tashi L. Peling ${ }^{3}$ and Andrew J. Worlock ${ }^{3}$

\begin{abstract}
Background: Quantitation of HIV-RNA is critically important for diagnosis, prognosis, treatment, monitoring and assessment of infectivity in HIV-1 infection. The objective of this study was to assess performance characteristics of the Aptima HIV-1 Quant Dx assay (Aptima), a new transcription mediated amplification (TMA), fully integrated and automated assay from Hologic Inc., San Diego, CA, USA.

The analytical sensitivity, analytical specificity, precision and detection of HIV-1 subtypes were tested based on commercially available international standards or panels. A selected group of 244 anti-HIV-1 (+) plasma samples was used for comparison with Roche COBAS Ampliprep/COBAS TaqMan HIV- 1 test v2.0 (Roche CAP/CTM), (Roche Molecular Systems, Pleasanton, CA).

Results: The 50 and $95 \%$ limit of detection were estimated at 4.9 (95\% Cl 3.9-5.7) and 17.6 (15.2-21.2) IU/mL respectively. The specificity was found $99.83(99.06-99.97) \%$. The standard deviations and coefficient of variations for panels with 50 and 100 copies $/ \mathrm{mL}$ (1.7 and 2 log copies $/ \mathrm{mL}$ ) were $0.14 \mathrm{log}$ copies $/ \mathrm{mL}(8.67 \% \mathrm{CV}$ ) and $0.18 \mathrm{log}$ copies $/ \mathrm{mL}$ (9.91\%CV) respectively. The detection rate for Aptima and Roche assays was 220/244 (90.2 \%) and 217/ 244 (88.9\%) respectively.
\end{abstract}

Conclusion: The Aptima assay is a sensitive, specific, precise and accurate test for measuring HIV-1 viral loads and for the detection of HIV-1 infections.

\section{Background}

Quantitation of HIV-RNA (viral load) has proven clinical utility in the diagnosis, prognosis, treatment, monitoring and the assessment of infectivity in HIV-1 infected patients [1-4].

HIV-1 is highly heterogeneous with Group M viruses responsible for the majority of HIV-1 infections worldwide. They are subdivided into nine subtypes (A-D, F-H, J, K) and numerous circulating recombinant forms (CRFs). Due

\footnotetext{
*Correspondence: ahatzak@med.uoa.gr

${ }^{1}$ Department of Hygiene, Epidemiology and Medical Statistics, Medical School, National and Kapodistrian University of Athens, Mikras Asias 75, GR-11527 Athens, Greece

${ }^{2}$ Hellenic Scientific Society for the Study of AIDS and Sexually Transmitted

Diseases, Athens, Greece

Full list of author information is available at the end of the article
}

to human movements and the recombination events the HIV-1 diversity is gradually increasing posing a challenge in the validity of viral load assays [5].

There are a number of commercially available viral load assays which are mainly based on real-time PCR [6-11]. These assays offer varying levels of automation based on dedicated hardware and software, including automated result interpretation as well as high throughput.

The Aptima HIV-1 Quant Dx assay (Hologic Inc. San Diego, CA, USA) is a fully integrated and automated assay for use on the Panther System. At present, the test is not approved for use in the US but is CE-IVD certified for the detection and quantitation of HIV-1. It utilizes transcription mediated amplification (TMA) involving target capture, target amplification by TMA and realtime detection of amplicons by fluorescent probes in a 
single tube. The Aptima HIV-1 Quant Dx assay requires only $0.5 \mathrm{~mL}$ of sample per reaction with a dead volume as little as $0.2 \mathrm{~mL}$.

The aim of this study was to assess the analytical performance characteristics of Aptima assay using commercially available panels and to evaluate it side-by-side with Roche CAP/CTM.

\section{Methods}

\section{Linearity}

Linearity of HIV-1 subtype B across the assay's dynamic range was assessed using AcroMetrix HIV-1 panel from Life Technologies (Catalog number 950470 batch number 400716). This panel has 1 HIV negative panel member and $6 \mathrm{HIV}-1$ positive panel members with concentrations ranging from $1 \mathrm{e} 2$ to $5 \mathrm{e} 6$ copies $/ \mathrm{mL}$. The recovered concentration of each panel member was compared to the concentration value provided by the vendor. The linearity of the various subtypes of HIV-1 was confirmed by testing 5-7 dilutions of clinical specimens (HIV subtypes A1, B, C, F1 and CRF02-AG) and cultured isolates of HIV (subtypes $\mathrm{G}$ and $\mathrm{A} / \mathrm{E}$ and group $\mathrm{O}$ and $\mathrm{N}$ ). The dilutions tested for clinical specimens targeted 1e2, 5e2, 1e3, 1e4 and 1e 5 copies $/ \mathrm{mL}$. The concentration and available volume of the clinical sample stocks did not permit testing concentrations above 1e 5 copies $/ \mathrm{mL}$. For the clinical isolates two additional dilutions at $1 \mathrm{e} 6$ copies $/ \mathrm{mL}$ and $5 \mathrm{e} 6$ copies $/ \mathrm{mL}$ were also tested.

\section{Analytical sensitivity (limit of detection)}

One vial of $2^{\text {nd }}$ WHO international standard for HIV-1 (NIBSC code 97/650) was reconstituted according to manufacturer's instructions. The sample was diluted in HIV-1 negative plasma to concentrations ranging from 0 to $40 \mathrm{IU} / \mathrm{mL}(0,5,10,15,20,25,30$ and $40 \mathrm{IU} / \mathrm{mL})$. Each panel member was tested on 3 Panthers with 3 reagent lots of Aptima HIV-1 assay reagent. Ten replicates were tested for each concentration with each reagent lot on each Panther (total 90 replicates per concentration). Probit analysis was performed with the Aptima results using SAS software to calculate the 50 and $95 \%$ detection rates.

\section{Analytical specificity}

Specificity was assessed by testing 600 plasma specimens collected from people who were not infected with HIV1. Testing was conducted with 2 reagent lots of Aptima assay reagents with 300 specimens being tested per reagent lot. Testing was distributed across 3 Panther instruments. All samples which gave reactive results $(<1.47 \log$ copies $/ \mathrm{mL}$ detected or quantitative result or $>7 \log$ copies $/ \mathrm{mL}$ ) were considered as false positives in Aptima.

\section{Precision, reproducibility and repeatability}

Within-run repeatability and between-run reproducibility of the Aptima assay was assessed by testing 8 dilutions of HIV-1 in negative plasma ranging in concentration from 50 to $10^{7}$ copies $/ \mathrm{mL}$. Each panel was tested on 3 Panthers with 3 Aptima reagent lots by three operators to a total of 27 runs. Three replicates of each panel were tested in each run. Results from all valid replicates were used for assessment of precision using SAS statistics software.

\section{Genetic variability and subtype detection}

Subtype detection was assessed using the German NRC subtype panel composed of 21 members of which 18 belong to group $M(2 x \mathrm{~A}, 2 \mathrm{x} B, 2 \mathrm{x}, 3 \mathrm{x} D, 2 \mathrm{x}$ CRF01_AE, 2x F, 1x G, 1x H, 1xG/H, 2x CRF02_AG), 2 to group $\mathrm{O}$ and 1 to group $\mathrm{N}$. These panels were manufactured by National Resource Center using virus isolates originally obtained from the NIH AIDS Research and Reference Reagent Program, USA, or from the Programme EVA Centre for AIDS Reagents, NIBSC, UK. These viruses were cultivated on cells and supernatants were diluted in HIV-negative plasma to a concentration of approximately 100,000 HIV-1 RNA copies/mL. The isolates were quantified using the Roche CAP/CTM and Abbott RealTime HIV-1 (Abbott RT) assays. One replicate of each subtype panel was tested in the Aptima assay and results were compared to the Roche CAP/ CTM and Abbott RT results provided by the vendor. This three-way comparison allowed for any results differing by more than $0.5 \log$ copies $/ \mathrm{mL}$ to be resolved.

\section{Assay comparison}

Assessment of agreement with an established commercially available method was undertaken (Roche CAP/ CTM). The Roche CAP/CTM assay targets the gag and LTR regions [12]. The assay uses at least $1 \mathrm{~mL}$ of specimen and reports quantifiable HIV-1 results over the range of 20 to 10,000,000 copies/mL [12]. The Aptima assay targets the pol and LTR regions. The assay uses $0.5 \mathrm{~mL}$ of specimen plus a minimum $0.2 \mathrm{ml}$ dead volume and reports quantifiable HIV-1 results over the range of 30 to 10,000,000 copies/mL [13]. Both Aptima and Roche assays report specificity of $100 \%$ in their respective package inserts $[12,13]$. Two hundred fortyfour patient plasma anti-HIV-1 (+) samples were collected, aliquoted and frozen. Samples were selected based on an historical HIV viral load result based on Roche AmpliPrep/COBAS Taqman HIV-1 to provide results across the range of both Roche and Aptima assays. One replicate of each sample was tested in Aptima and Roche assays following the manufacturer's instructions. One hundred seventy-four samples which had quantitative results in both assays were used for linear 
regression and Bland-Altman analysis. These included different HIV-1 subtypes and recombinants.

\section{Statistical methods}

The 50 and $95 \%$ positivity rates were estimated using Probit analysis using the normal model and analysis software from the SAS Institute Cary, NC, USA.

The method comparison simple linear regression and Bland Altman analysis were performed using Analyse-it Software, Leeds, United Kingdom.

Comparison of the Aptima HIV-1 results to Roche CAP/CTM was performed using McNamara's test with analysis software from the SAS Institute Cary, NC, USA.

Linearity was measured using the $\mathrm{R}^{2}$ of the Pearson correlation coefficient calculated using Microsoft Excel (WA, USA).

\section{Results}

Linearity

As seen from Table 1 all members of the HIV-1 subtype B AcroMetrix linearity panel, ranging in concentration from $1 \mathrm{e} 2$ to $5 \mathrm{e} 6$ copies $/ \mathrm{mL}$, recovered within $0.25 \operatorname{logs}$ of the target concentration. The results in Aptima were also linear for all the major groups, subtypes and recombinants of HIV (Figs. 1 and 2). The $\mathrm{R}^{2}$ values of the plots in Figs. 1 and 2 were all above 0.999 as shown in Table 2 .

\section{Analytical sensitivity of Aptima}

By use of HIV-1 $2^{\text {nd }}$ International WHO standards diluted in plasma, the 50 and $95 \%$ limit of detection and $95 \%$ confidence intervals (shown in brackets) were estimated by Probit analysis as $4.9(3.9-5.7)$ and 17.6 (15.2-21.2) IU/mL, respectively (Table 3 ).

\section{Analytical specificity}

The combined analytical specificity using 600 HIV-1 negative specimens from two different lots was estimated 99.83 (95 \% CI of 99.06-99.97\%) (Table 4).

Table 1 Recovery of AcroMetrix subtype B Linearity Panel in Aptima HIV Quant Dx

\begin{tabular}{lll}
\hline $\begin{array}{l}\text { Target concentration } \\
\text { (log copies } / \mathrm{mL})\end{array}$ & $\begin{array}{l}\text { Average Aptima } \\
\text { (log copies } / \mathrm{mL})\end{array}$ & $\begin{array}{l}\text { Average Aptima- } \\
\text { Target }\end{array}$ \\
\hline Not detected & Not detected & Not detected \\
2.00 & 2.05 & 0.05 \\
2.70 & 2.60 & -0.10 \\
3.70 & 3.65 & -0.05 \\
4.70 & 4.86 & 0.16 \\
5.70 & 5.93 & 0.23 \\
6.70 & 6.92 & 0.22 \\
\hline
\end{tabular}

\section{Precision, reproducibility and repeatability}

The CVs for inter-instrument, inter-operator, inter-lot, inter-day, inter-run and intra-run variation were 0 $4.52 \%, 0-1.99 \%, 0-0.77 \%, 0-0.35 \%, 0-5.6 \%$ and $0.44-8.14 \%$, respectively. The total CVs for panels of 50 copies $/ \mathrm{mL}, 10^{2}, 10^{3}, 10^{4}, 10^{5}, 10^{6}, 10^{7}$ copies $/ \mathrm{mL}$ were estimated $8.67 \%, 9.91 \%, 4.08 \%, 1.77 \%, 1.35 \%, 0.92 \%$, $0.9 \%$ respectively (Table 5 ).

\section{Genetic variability and subtype detection using HIV-1 viral isolates}

Twenty one viral isolates were used for comparison of Aptima Roche CAP/CTM and Abbott RT. Aptima consistently quantified slightly higher compared to target values based on Roche CAP/CTM with differences ranging from 0.01 to $0.79 \log$ copies $/ \mathrm{mL}$ (Table 6). The differences between Aptima and Abbott RT results ranged from -0.12 to $1.04 \log$ copies $/ \mathrm{mL}$.

\section{Comparison of Aptima with Roche CAP/CTM using clinical samples}

Aptima quantified 220/244 (90.1 \%) and Roche 217/244 $(88.9 \%)$ of the clinical samples. Eleven samples were Roche (+), Aptima (-) and 14 samples Aptima (+), Roche $(-)$ (Table 7). One hundred seventy four samples were quantified by both methods (McNemar's $p=0.5485$ ).

Linear regression analysis showed excellent correlation between Aptima and Roche $\left(R^{2}=0.971\right)$. The slope was 1.06 (Fig. 3).which was statistically significant ( $p$-value < $0.0001)$. The intercept was -0.034 which was not statistically significant ( $p$-value 0.566 ). By Bland-Altman analysis (Fig. 4), the bias was $0.212 \log$ copies $/ \mathrm{mL}$ with $95 \%$ agreement intervals of -0.259 to 0.683 .

Forty seven of 174 quantifiable by both assays were subtype B, 99 subtype A, 11 Subtype C, 3 subtype G and 14 were CRFs.

Linear regression for subtype B is shown in Fig. 5. The $\mathrm{R}^{2}$ was 0.968 . The slope was 1.02 (Fig. 5) which was statistically not significant ( $p$-value 0.384 ). The intercept was 0.08 which was not statistically significant ( $p$-value 0.494). By Bland-Altman analysis (Fig. 6) the bias was $0.179 \log$ copies $/ \mathrm{mL}$ with $95 \%$ agreement intervals of -0.258 to 0.617 .

Linear regression for non-B subtypes is shown in Fig. 7. The $\mathrm{R}^{2}$ was 0.972 . The slope was 1.07 (Fig. 7) which was statistically significant ( $p$-value $<0.0001)$. The intercept was -0.07 which was not statistically significant ( $p$-value 0.327). By Bland-Altman analysis (Fig. 8) the bias was $0.179 \log$ copies $/ \mathrm{mL}$ with $95 \%$ agreement intervals of -0.257 to 0.706 .

\section{Discussion}

Detection and quantification of HIV-1 is important not only for diagnosis of HIV-1 infections but also for 


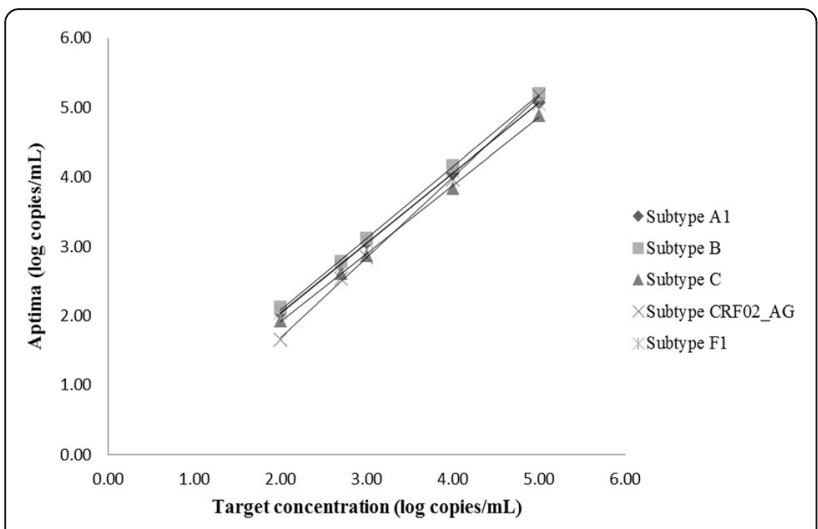

Fig. 1 Linearity of Aptima based on clinical specimens representing different HIV subtypes

management of HIV-1 patients [14]. High viral loads are correlated with an increased risk of clinical progression of HIV-associated disease, and reductions in plasma virus levels may be associated with decreased risk of clinical progression [15-17]. To ensure the best management of HIV positive patients it is important that the test used to measure HIV-1 viral loads is sensitive, specific, accurate and precise. As shown in this study the Aptima assay on the Panther System is a highly sensitive, specific and reproducible test for the detection and quantitation of HIV-RNA across a wide dynamic range.

As evident from the results of the AcroMetrix linearity panel (Table 1) and dilutions of HIV subtype clinical samples and isolates (Figs. 1 and 2) good linearity was demonstrated across the assay range for all subtypes of HIV in Aptima. The $\mathrm{R}^{2}$ values for all the HIV groups and subtypes tested were 0.999 or greater as shown in Table 2. The HIV subtypes included A, B, C, AG, F1, $\mathrm{AE}$ and $\mathrm{G}$ together with groups $\mathrm{N}$ and $\mathrm{O}$. This shows that the Aptima assay remains linear across a genetically diverse sample population. This confirms the results

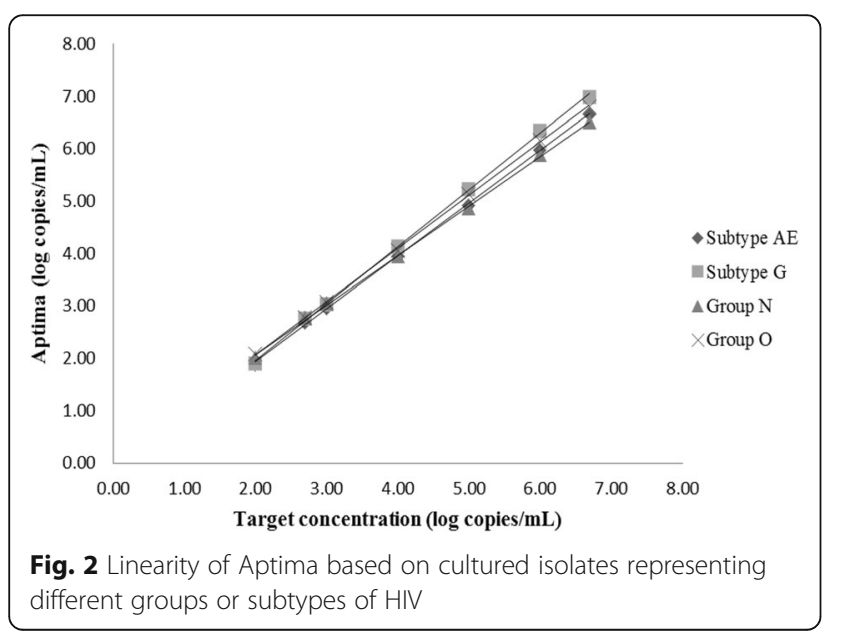

Table $2 R^{2}$ Values Linearity of Plots Aptima Based on Clinical Specimens Representing Different HIV Groups or Subtypes

\begin{tabular}{ll}
\hline HIV Group or Subtype & $R^{2}$ \\
\hline A & 1.0000 \\
B & 0.9996 \\
C & 0.9995 \\
AG & 0.9996 \\
F1 & 0.9995 \\
AE & 0.9996 \\
G & 0.9994 \\
N & 0.9994 \\
O & 0.9997 \\
\hline
\end{tabular}

shown in the Aptima package insert [13] for HIV-1 groups $\mathrm{M}, \mathrm{N}$ and $\mathrm{O}$.

The data presented in the package inserts for Roche CAP/CTM [12] and Aptima [13] show the assays are standardized to two different WHO standards (the $2^{\text {nd }}$ and $3^{\text {rd }}$ lot of the WHO standard respectively). To enable a direct comparison of analytical sensitivity between the two assays, the data on analytical sensitivity presented in this paper was based on dilutions of the $2^{\text {nd }}$ WHO international standard run in the Aptima assay. The data is presented in $\mathrm{IU} / \mathrm{mL}$ for this analysis to avoid any issues converting between $\mathrm{IU} / \mathrm{mL}$ to copies $/ \mathrm{mL}$ for assays standardized using two different WHO standards. The $95 \%$ limit of detection (LoD) of Aptima was found to be 17.6 (95 \% CI 15.2-21.2) IU/mL. This is slightly more sensitive than the reported value for Roche CAP/ CTM assay at $33 \mathrm{IU} / \mathrm{mL}[12,18]$. The higher sensitivity of Aptima compared to Roche CAP/CTM has been reported by other investigators [19]. Table 7 shows 14 Aptima positive, Roche CAP/CTM negative results compared to 11 Aptima negative, Roche CAP/CTM positive results. Although this was not statistically significant it suggests the Aptima assay is more sensitive than the

Table 3 Assessment of analytical sensitivity of Aptima HIV-1 Quant by dilutions of HIV-1 2nd international WHO standard diluted in plasma

\begin{tabular}{llll}
\hline Panels IU/mL & Number & Number positive & $\%$ Positive \\
\hline 0 & 89 & 0 & $0 \%$ \\
5 & 89 & 46 & $52 \%$ \\
10 & 90 & 73 & $81 \%$ \\
15 & 90 & 84 & $93 \%$ \\
20 & 90 & 88 & $98 \%$ \\
25 & 89 & 87 & $98 \%$ \\
30 & 90 & 89 & $99 \%$ \\
40 & 89 & 89 & $100 \%$ \\
\hline
\end{tabular}


Table 4 Assessment of analytical specificity of Aptima HIV-1 Quant Dx assay by two lots of HIV-1 negative samples

\begin{tabular}{llllll}
\hline Reagent lot & $\mathrm{N}$ & Negative & Specificity & $\mathrm{LCl}$ & $\mathrm{UCl}$ \\
\hline lot 1 & 300 & 299 & 99.67 & 98.14 & 99.94 \\
lot 2 & 300 & 300 & 100 & 98.74 & 100.00 \\
Combined & 600 & 599 & 99.83 & 99.06 & 99.97
\end{tabular}

Roche CAP/CTM. The high sensitivity of the Aptima assay indicates that it will be useful for both as an aid in clinical management of and diagnosing patients with HIV-1 infections.

It is also important for effective patient management that the specificity of the assay is high. In this study specificity was $99.83 \%$ when testing 600 plasma samples. A single sample from 600 gave a positive result. The positive result was reported as " $<30$ copies/mL detected" and so was below the lower limit of quantitation for the assay. Since the result was not quantifiable it represents a very low level of HIV-1 between 1 and 30 copies $/ \mathrm{mL}$.

The subtype detection was evaluated using the NRC subtype panel composed of 21 panel members targeted at approximately 100,000 HIV-1 RNA copies/mL. The isolates included subtype A, B, C, D, F, G and H as well as circulating recombinants. Viral load results for the Aptima assay were slightly higher than those for Roche CAP/CTM but were within $0.5 \log$ copies $/ \mathrm{mL}$ in all cases with one exception. This isolate which was a recombinant of $\mathrm{G}$ and $\mathrm{H}$ had a viral load result from Aptima that was $0.79 \log$ copies $/ \mathrm{mL}$ higher than Roche CAP/CTM. Abbott RT results were obtained for this panel member which allow a comparison to be made to a third assay. The Aptima result was within $0.27 \operatorname{logs}$ of the Abbott RT result. This suggests that the Roche
CAP/CTM result is low. Results for the remaining panel members were available from the vendor for the Abbott RT assay. The Aptima results were within 0.5 logs of the Abbott RT result for 19 of the 21 samples. For 2 samples the Abbott RT results were 0.51 (subtype $\mathrm{H}$ ) and 1.04 (Group N) logs lower than Aptima. The Aptima result was within 0.5 logs of the Roche CAP/CTM result for the Group N sample suggesting that the Abbott RT result is low. Roche CAP/CTM results were unavailable for the subtype $\mathrm{H}$ sample. This data confirms the results in the Aptima package insert [13] showing equal HIV-1 group and subtype quantitation.

In the head to head comparison of Aptima and Roche CAP/CTM high correlation was seen between the results in the two assays. The slope was 1.06 for all quantifiable results. The Aptima assay gave slightly higher results compared to Roche CAP/CTM at the higher range of the assay and this contributed to the slope. These differences are not clinically significant and are similar to other studies [20, 21]. The average bias between Aptima and Roche CAP/CTM was $0.212 \mathrm{log}$ copies/mL which was mainly driven by higher Aptima values at the higher range of the assay. This small bias is not clinically significant. HIV subtype B is the most prevalent subtype in Western Europe [22]. The clinical specimen data set was broken into two subsets for further analysis (subtype B samples and non subtype B samples). Compared to Roche CAP/CTM, the Aptima assay had a slope of 1.02 for subtype $B$ and 1.07 for nonsubtype B samples. The Bland Altman plot showed a slight positive bias for this data of $0.179 \log$ copies $/ \mathrm{mL}$ for both sets of data but this is not clinically significant. The higher slope seen for non subtype B compared to subtype $B$ suggests that the high proportion of non $B$ subtypes in the overall comparison to Roche CAP/CTM contributed to the higher slope. In general the results in

Table 5 Assessment of inter-instrument, inter-operator, inter-lot, inter-day, inter-run, intra-run and total variability of Aptima HIV-1 Quant Dx in various HIV-1 RNA concentrations

\begin{tabular}{|c|c|c|c|c|c|c|c|c|c|c|c|c|c|c|c|c|}
\hline \multirow[b]{2}{*}{ Panel } & \multirow[b]{2}{*}{$\mathrm{N}$} & \multirow[b]{2}{*}{$\begin{array}{l}\text { Mean } \\
\text { Log }\end{array}$} & \multicolumn{2}{|c|}{ Inter-instrument } & \multicolumn{2}{|c|}{ Inter-operator } & \multicolumn{2}{|c|}{ Inter-lot } & \multicolumn{2}{|c|}{ Inter-day } & \multicolumn{2}{|c|}{ Inter-run } & \multicolumn{2}{|c|}{ Intra-run } & \multicolumn{2}{|l|}{ Total } \\
\hline & & & $\overline{S D}$ & $\begin{array}{l}\mathrm{CV} \\
(\%)\end{array}$ & $\overline{S D}$ & $\begin{array}{l}C V \\
(\%)\end{array}$ & $\overline{S D}$ & $\begin{array}{l}C V \\
(\%)\end{array}$ & SD & $\begin{array}{l}C V \\
(\%)\end{array}$ & $\overline{S D}$ & $\begin{array}{l}C V \\
(\%)\end{array}$ & $\overline{S D}$ & $\begin{array}{l}C V \\
(\%)\end{array}$ & $\overline{S D}$ & $\begin{array}{l}\mathrm{CV} \\
(\%)\end{array}$ \\
\hline 5E1 copies/mL & $41^{a}$ & 1.66 & 0.075 & 4.52 & 0.033 & 1.99 & 0 & 0 & 0 & 0 & 0 & 0 & 0.119 & 7.12 & 0.144 & 8.67 \\
\hline $1 \mathrm{E} 2$ copies/mL & $74^{\mathrm{a}}$ & 1.82 & 0 & 0 & 0 & 0 & 0.014 & 0.77 & 0 & 0 & 0.102 & 5.6 & 0.148 & 8.14 & 0.18 & 9.91 \\
\hline 1E3 copies/mL & 81 & 2.75 & 0.035 & 1.26 & 0 & 0 & 0 & 0 & 0 & 0 & 0.041 & 1.5 & 0.098 & 3.57 & 0.112 & 4.08 \\
\hline $1 \mathrm{E} 4$ copies/mL & 81 & 3.81 & 0.011 & 0.28 & 0.013 & 0.35 & 0 & 0 & 0.013 & 0.35 & 0.05 & 1.31 & 0.039 & 1.04 & 0.067 & 1.77 \\
\hline 1E5 copies/mL & 81 & 4.96 & 0.01 & 0.21 & 0 & 0 & 0 & 0 & 0 & 0 & 0.042 & 0.84 & 0.051 & 1.03 & 0.067 & 1.35 \\
\hline 1 E6 copies/mL & $78^{b}$ & 6 & 0.008 & 0.14 & 0.001 & 0.02 & 0 & 0 & 0 & 0 & 0.048 & 0.79 & 0.027 & 0.44 & 0.055 & 0.92 \\
\hline 1E7 copies/mL & 81 & 6.89 & 0 & 0 & 0 & 0 & 0.026 & 0.38 & 0 & 0 & 0.033 & 0.47 & 0.046 & 0.67 & 0.062 & 0.9 \\
\hline
\end{tabular}

Note: 1 . Variability from some factors may be numerically negative, which can occur if the variability due to those factors is very small. When this occurs, SD $=0$ and $\mathrm{CV}=0 \%$

athe 50 and 100 copies $/ \mathrm{mL}$ sample has lower $\mathrm{N}$ because some results were below the limit of quantification

$b_{3}$ results were invalid and not included in the analysis 
Table 6 Subtype detection by aptima HIV-1 quant and roche CAP/CTM in viral isolates diluted to $10^{5}$ copies/mL of HIV-RNA

\begin{tabular}{|c|c|c|c|c|c|c|}
\hline Isolate & $\begin{array}{l}\text { Subtype/ } \\
\text { Group }\end{array}$ & $\begin{array}{l}\text { Aptima log Copies/ } \\
\mathrm{mL}\end{array}$ & $\begin{array}{l}\text { Roche CAP/CTM log } \\
\text { Copies/mL }\end{array}$ & $\begin{array}{l}\text { Aptima - Roche CAP/ } \\
\text { CTM }\end{array}$ & $\begin{array}{l}\text { Abbott RT (Log Copies/ } \\
\mathrm{mL} \text { ) }\end{array}$ & $\begin{array}{l}\text { Aptima- Abbott } \\
\text { RT }\end{array}$ \\
\hline$\overline{92 \cup G 029}$ & $\mathrm{~A}$ & 5.92 & 5.57 & 0.35 & 5.60 & 0.32 \\
\hline 00KE_KER2018 & A & 5.11 & 4.98 & 0.13 & 5.02 & 0.09 \\
\hline 92TH026 & B & 5.54 & 5.49 & 0.06 & 5.66 & -0.12 \\
\hline 90TH_BK132 & B & 5.57 & 5.37 & 0.21 & 5.36 & 0.21 \\
\hline 92BR025 & C & 5.51 & 5.26 & 0.25 & 5.32 & 0.18 \\
\hline 99ET_14 & C & 6.38 & 6.12 & 0.26 & 5.91 & 0.48 \\
\hline $92 \cup G 021$ & D & 5.47 & 5.45 & 0.03 & 5.51 & -0.03 \\
\hline 92UG035 & D & 6.02 & 5.61 & 0.40 & 5.77 & 0.25 \\
\hline $92 \cup G 024$ & D & 5.74 & 5.43 & 0.30 & 5.61 & 0.13 \\
\hline 92ТH022 & CRF01_AE & 5.25 & not available & not available & 5.26 & -0.01 \\
\hline 92TH009 & CRF01_AE & 5.33 & 5.08 & 0.25 & 5.25 & 0.08 \\
\hline 93BR029 & $\mathrm{F}$ & 5.15 & 5.16 & 0.01 & 5.18 & -0.03 \\
\hline 93BR020 & $\mathrm{F}$ & 5.59 & not available & not available & 5.19 & 0.40 \\
\hline RU570 & G & 5.77 & not available & not available & 5.70 & 0.08 \\
\hline V1557 & $\mathrm{H}$ & 5.95 & not available & not available & 5.43 & 0.51 \\
\hline 01CM.0005BBY & CRF02_AG & 4.89 & 4.77 & 0.12 & 4.68 & 0.21 \\
\hline 01CM.0008BBY & CRF02_AG & 5.23 & 5.00 & 0.23 & 4.95 & 0.27 \\
\hline VI525 & $\mathrm{G} / \mathrm{H}$ & 5.21 & 4.42 & 0.79 & 4.94 & 0.27 \\
\hline YBF 30 & N & 6.20 & 5.72 & 0.48 & 5.17 & 1.04 \\
\hline MVP5180 & $\mathrm{O}$ & 4.32 & not available & not available & 4.23 & 0.09 \\
\hline CA-9 & $\mathrm{O}$ & 4.76 & 4.75 & 0.01 & 4.32 & 0.43 \\
\hline
\end{tabular}

this study are favorably compared with previous comparisons with the Aptima assay and assays such as Abbott RealTime, Roche CAP/CTM, COBAS ${ }^{\bullet}$ TaqMan $^{\circ}$ HIV-1 Test v2.0 for use with the High Pure System (HPS/ CTM), Artus HIV-1 on the Qiagen Rotor-Gene Q and Nuclisens EasyQ v2 (BioMerieux SA, Marcy L'Etoile, France) [13, 23-25].

There are significant advantages when running the Aptima assay for lab work flow. The Panther system is fully automated so that samples can be placed directly on the system without prior nucleic acid extraction. This system allows random access testing of various analytes, processing up to 320 samples in an 8-hour shift and

Table 7 Qualitative $2 \times 2$ comparison of Aptima and Roche CAP/CTM

\begin{tabular}{cllll}
\hline & \multicolumn{4}{c}{ Aptima } \\
\cline { 2 - 5 } & & + & - & Total \\
\hline Roche CAP/CTM & + & 206 & 11 & 217 \\
& - & 14 & 13 & 27 \\
& Total & 220 & 24 & 244
\end{tabular}

The $p$-value for McNemar's test is 0.5485 indicating that the difference in positivity between Aptima and Roche for the type of samples tested is not statistically significant

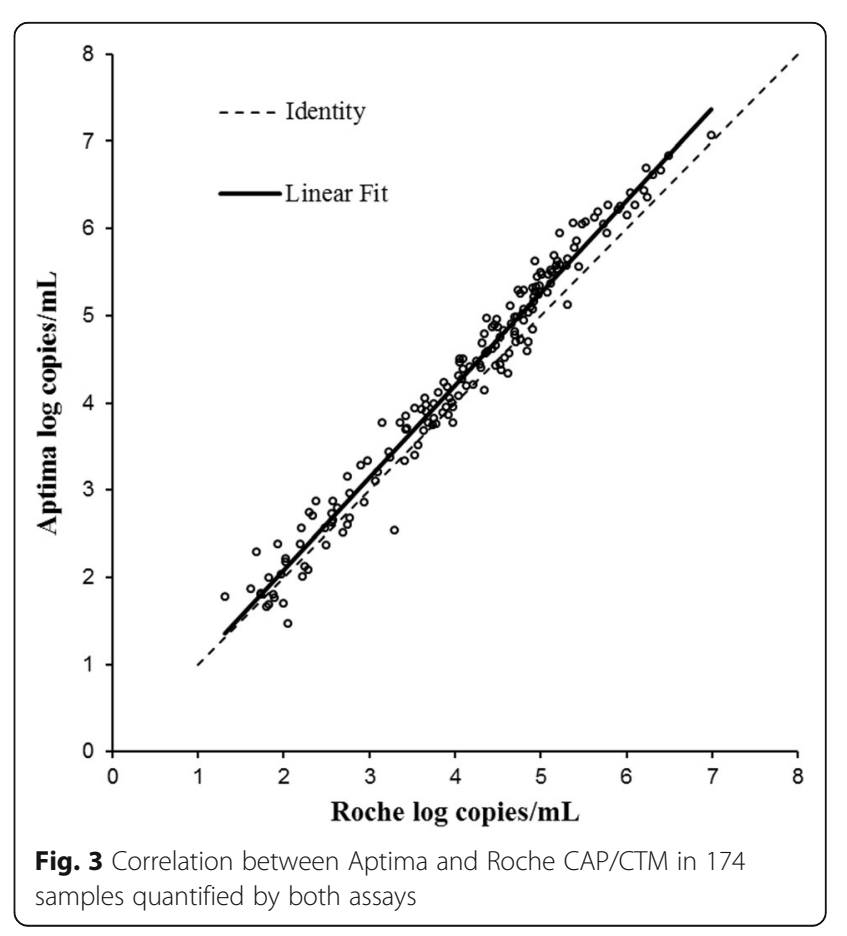




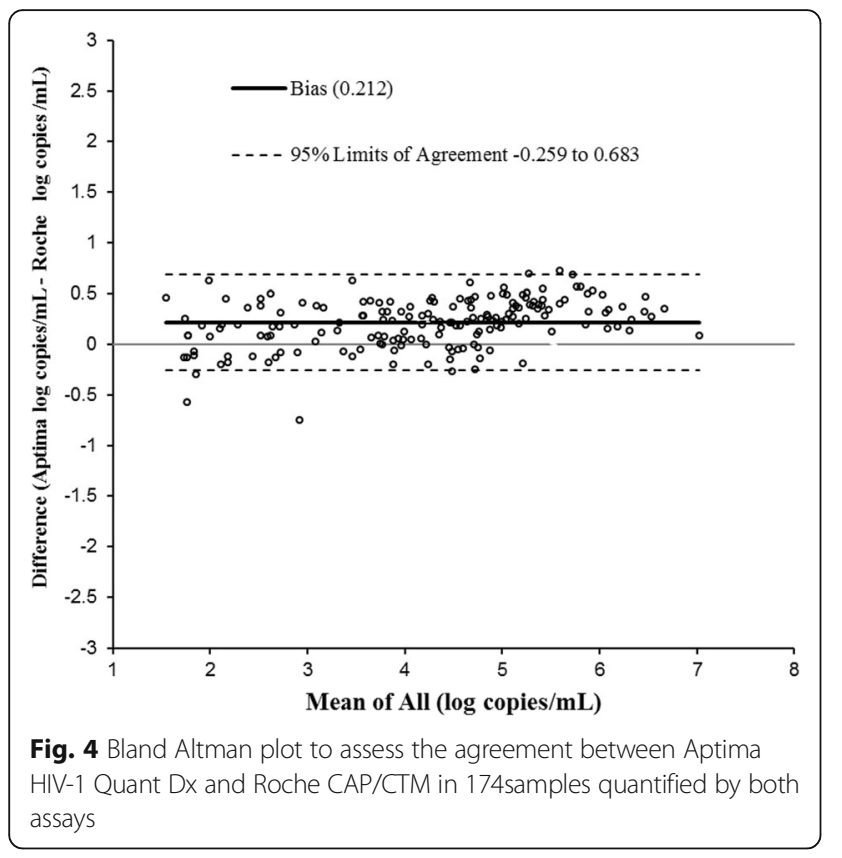

returning of results in about 2.5 hours. This enables high flexibility to adapt to low or high throughput testing. In addition, as reported by Sam et al. [21] the cumulative hands-on time required for specimen processing and maintenance steps were consistently shorter with the Panther than with the Abbott m2000 system.

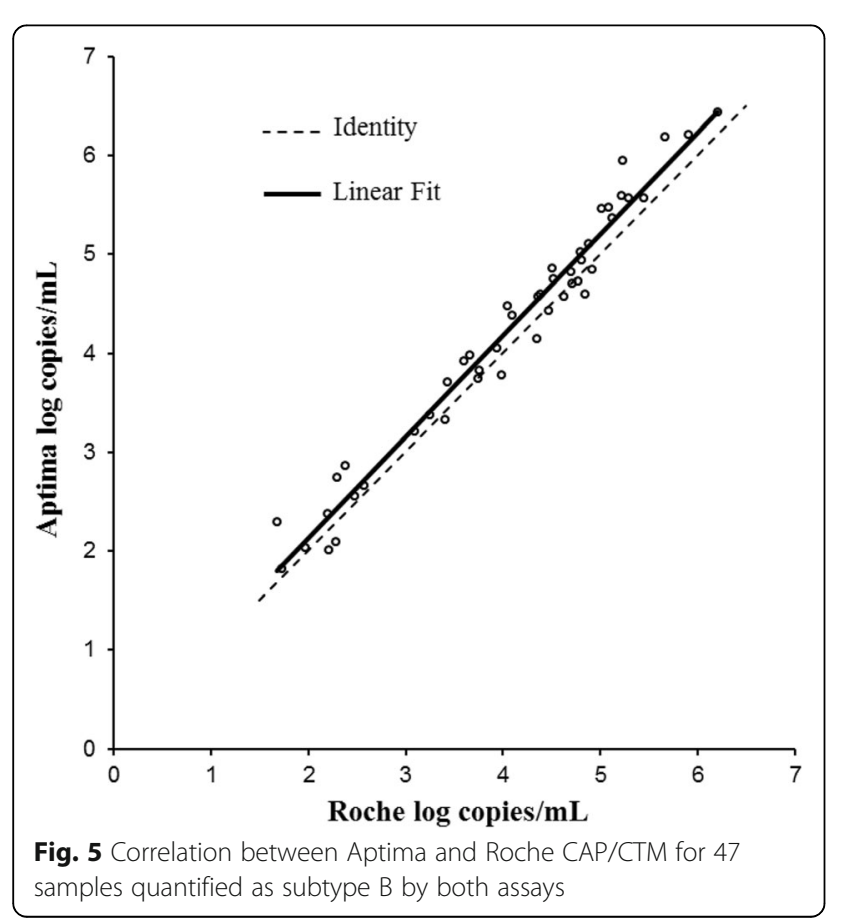

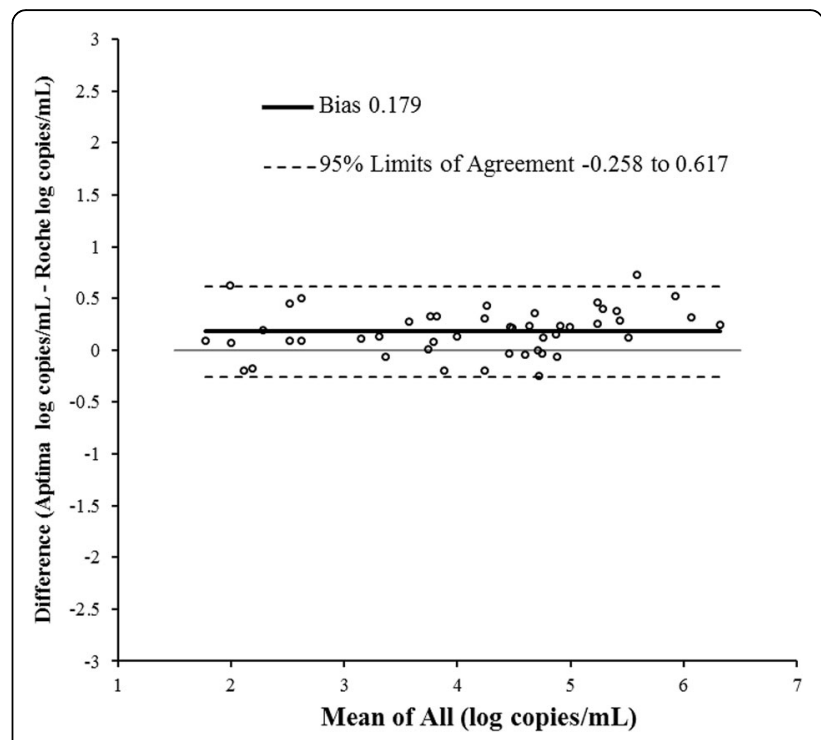

Fig. 6 Bland Altman plot to assess the agreement between Aptima and Roche CAP/CTM in 47 subtype B samples quantified by both assays

\section{Conclusion}

In conclusion, the Aptima assay has excellent comparative performance with Roche CAP/CTM. The assay is a good choice for clinicians in Europe for the detection and monitoring of patients infected with HIV-1 as it has CE-IVD certification. At present, the test is not approved for use in the US. The high sensitivity along with

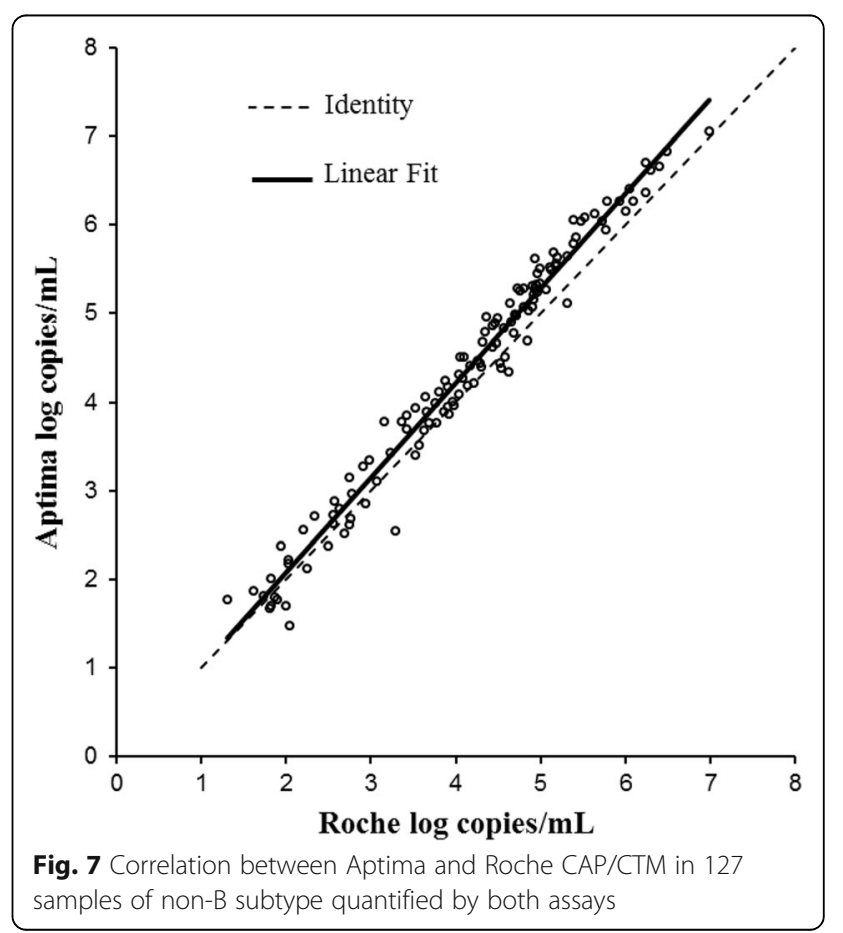




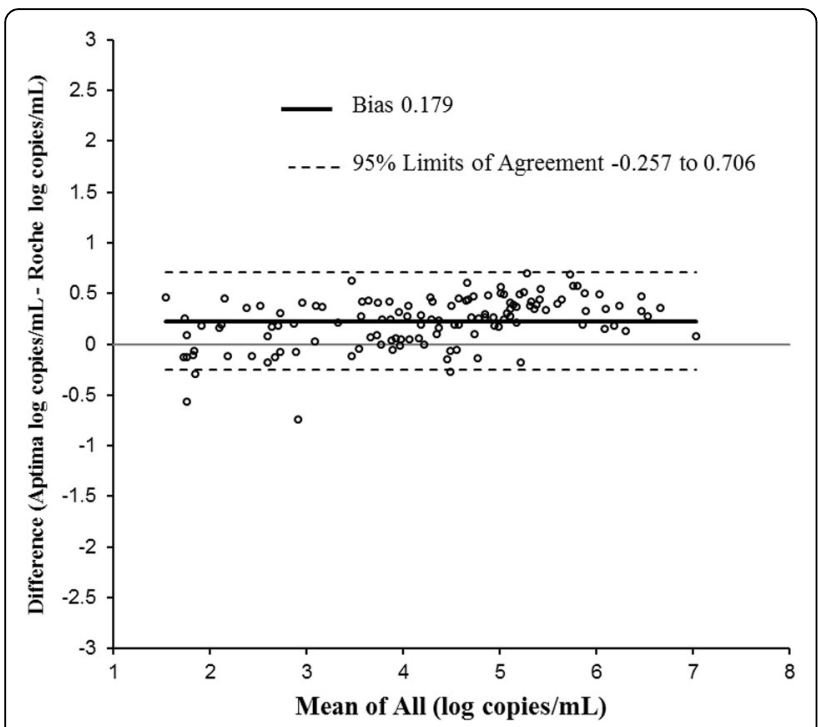

Fig. 8 Bland Altman plot to assess the agreement between Aptima HIV-1 and Roche CAP/CTM in 127 samples of non-B subtypes quantified by both assays

close agreement to Roche CAP/CTM suggests rebaseline of patients is not required when switching to the Aptima assay for HIV-1 testing.

\section{Abbreviations}

Abbott RT: Abbott RealTime; AIDS: Acquired immune deficiency syndrome; Aptima Assay: Aptima HIV-1 Quant Dx assay; CRF: Circulating recombinant forms; CV: Coefficient of variation; HIV: Human immunodeficiency virus; LoD: Limit of detection; mL: Millilitre; NIBSC: National Institute for Biological Standards and Control; NRC: National Reference Centre; PCR: Polymerase chain reaction; RNA: Ribonucleic acid; Roche CAP/CTM: COBAS Ampliprep/ COBAS TaqMan HIV-1 test v2.0; TMA: Transcription mediate amplification; WHO: World Health Organization

\section{Acknowledgements}

Youna Kang from Hologic Inc. helped create panels for the linearity study.

\section{Availability of data and materials}

Supplemental Material is not available.

\section{Authors' contributions}

AH contributed to writing the manuscript and helped procure the clinical specimens used in the study. HP procured the clinical specimens used in the study and generated the Roche CAP/CTM results. SJN analyzed the Aptima results and prepared samples for Aptima testing. JF tested samples using the Aptima assay and also analyzed results. TF tested samples using the Aptima assay and also analyzed results. HCK helped with sample testing and analyzed results. TLP tested Acrometrix and NRC panels using the Aptima assay. AJW contributed to writing the manuscript and analyzing the results. All authors read and approved the final manuscript.

\section{Competing interests}

$\mathrm{AH}$ and HP declare that they have no competing interests.

SJN, JF, TF, HCK, TLP and AJW are employees of Hologic.

\section{Ethics approval and consent to participate}

Patient informed consent is not required since the data were collected from investigational testing, they were anonymized and cannot be linked to the individuals. The study was approved by the IRB of the Hellenic Scientific Society for the Study of AIDS and Sexually Transmitted Diseases.

\section{Author details}

'Department of Hygiene, Epidemiology and Medical Statistics, Medical School, National and Kapodistrian University of Athens, Mikras Asias 75, GR-11527 Athens, Greece. ${ }^{2}$ Hellenic Scientific Society for the Study of AIDS and Sexually Transmitted Diseases, Athens, Greece. ${ }^{3}$ Hologic Inc., 10210 Genetic Center Drive, San Diego, CA 92121, USA. ${ }^{4}$ Department of Hygiene, Epidemiology and Medical Statistics, National Retrovirus Reference Center, National and Kapodistrian University of Athens Medical School, Mikras Asias 75, GR-11527 Athens, Greece.

Received: 16 June 2016 Accepted: 29 September 2016

Published online: 21 October 2016

\section{References}

1. Ho DD, Neuman AU, Perelson AS, Chem W, Leonard JM, Markowitz M. Rapid turnover of plasma virions and CD4 Lymphocytes in HIV-1 infection. Nature. 1995;373:123-6.

2. Mellors JW, Muñoz A, Giorgi JV, Margolick JB, Tassoni CJ, Gupta P, Kingsley LA, Todd JA, Saah AJ, Detels R, Phair JP, Rinaldo Jr CR. Plasma viral load and CD4+ lymphocytes as prognostic markers of HIV-1 infections. Ann Intern Med. 1997;126:946-54.

3. O'Brien TR, Blatner WA, Waters D, Eyster E, Hilgartner MW, Cohen AR, Luban M, Hatzakis A, Aledort LM, Rosenberg PS, Miley WJ, Kroner BL, Goedert JJ. Serum HIV-1 RNA levels and time to development of AIDS in the multicenter hemophilia cohort study. JAMA. 1996;276:105-10.

4. European AIDS Clinical Society (EACS), Guidelines, Version 8.0, October 2015 http://www.eacsociety.org/files/2015_eacsguidelines_8.0-english_rev20151221.pdf. Accessed 3 Oct 2016.

5. Paraskevis D, Magiorkinis E, Magiorkinis G, Sypsa V, Paparizos V, Lazanas M, Gargalianos P, Antoniadou A, Panos G, Chrysos G, Sambatakou H, Karafoulidou A, Skoutelis A, Kordossis T, Koratzanis G, Theodoridou M, Daikos GL, Nikolopoulos G, Pybus OG, Hatzakis A. Increasing Prevalence of HIV-1 Subtype A in Greece: Estimating Epidemic History and Origin. J Infect Dis. 2007;196:1167-76

6. Katsoulidou A, Rokka C, Issaris C, Haida C. Tzannis K, Sypsa V, Detsika M, Paraskevis D, Hatzakis A. Comparative evaluation of the performance of the Abbott RealTime HIV-1 assay for measurement of HIV-1 plasma viral load on genetically diverse samples from Greece. Virol J. 2011;8:10.

7. Abbott Molecular RealTime HIV-1 [product information]. Available at: http:// www.abbottmolecular.com/products/infectious-diseases/realtime-pcr/hiv-1assay. Accessed 3 Oct 2016.

8. Braun $P$, Ehret $R$, Wiesmann F, Braun $P$, Ehret $R$, Wiesmann F, Zabbai F, Knickmann M, Kühn R, Thamm S, Warnat G, Knechten H. Comparison of four commercial quantitative HIV-1 assays for VL monitoring in clinical daily routine. Clin Chem Lab Med. 2007;45:93-9.

9. Saune K, Delaquerre C, Raymod S, Nicot F, Boineau J, Pasquier C, Izopet J. Analytical sensitivity of three real-time PCR assays for measuring subtype B HIV-1 RNA. J Clin Virol. 2013:57:80-3.

10. Schutten M, Peters D, Back NK, Beld M, Beuselinck K, Foulongne V, Geretti A, Pandiani L, Tiemann C, Niesters HGM. Multicenter evaluation of the new Abbott RealTime assays for quantitative detection of human immunodeficiency virus type 1 and hepatitis C virus RNA. J Clin Microbiol. 2007:45:1712-7

11. Garcia-Diaz A, Clewley GS, Booth CL, Labett W, McAllister N, Geretti AM. Comparative evaluation of the performance of the Abbott real-time human immunodeficiency virus type 1 (HIV-1) assay for the measurement of HIV-1 plasma viral load following automated specimen preparation. J Clin Microbiol. 2006;44(5):1788-91.

12. COBAS AmpliPrep/COBAS Tagman HIV-1 Test version 2.0 Package insert 05328276001-01EN. https://molecular.roche.com/assays/cobas-ampliprepcobas-taqman-hiv-1-test-v2.

13. Aptima HIV-1 Quant Dx Assay Hologic Package insert AW-11853-001 Rev. 002. Available at: http://stage.hologic.com/products/clinical-diagnostics-andblood-screening/assays-and-tests/Aptima-hiv-1-quant-dx-quant-dx-assay.

14. Mellors JW, Rinaldo Jr CR, Gupta P, White RM, Todd JA, Kingsley LA. Prognosis in HIV-1 infection predicted by the quantity of virus in plasma. Science. 1996;272:1167-70

15. O'Brien WA, Hartigan PM, Martin D, Esinhart t, Hill A, Benoit S, Rubin M, Simberkoff MS, Hamilton JD. Changes in plasma HIV-1 RNA and CD4 lymphocyte counts and the risk of progression to AIDS. Veterans Affairs Cooperative Study Group on AIDS. N Engl J Med. 1996;334:426-31. 
16. Welles $S L$, Jackson JB, Yen-Lieberman B, Demeter L, Japour AJ, Smeaton LM, Johnson VA, Kuritzkes DR, Reichelderfer RTPA, Richman DD, Reichman R, Fischl M, Dolin R, Coombs RW, Kahn JO, McLaren C, Todd J, Kwok S, Crumpacker CS. Prognostic value of plasma human immunodeficiency virus type I (HIV-1) RNA levels in patients with advanced HIV-1 disease and with little or no zidovudine therapy. AIDS Clinical Trials Group Protocol 116A 116B/117 Team. J Infect Dis. 1996;174:696-703.

17. Coombs RW, Welles SL, Hooper C, Reichelderfer PS, D'Aquila RT, Japour AJ, Johnson VA, Kuritzkes DR, Richman DD, Kwok S, Todd J, Jackson JB, DeGruttola V, Crumpacker CS, Kahn J. Association of plasma human immunodeficiency virus Type I RNA level with risk of clinical progression in patients with advanced infection. AIDS Clinical Trials Group (ACTG) 116B/ 117 Study Team. ACTG Virology Committee Resistance and HIV-1 RNA Working Groups. J Infect Dis. 1996;174:704-12.

18. van Rensburg EJ, Tait K, Watt A, Schall R. Comparative Evaluation of the Roche Cobas AmpliPrep/Cobas TaqMan HIV-1 Version 2 Test Using the TaqMan 48 Analyzer and the Abbott RealTime HIV-1 Assay. J Clin Microbiol. 2011:49:377-9.

19. O'Shea S, Vijaysri Nair S, Kim H, Nugent CT, Yan C, Tong W, Douthwaite S, Worlock A. Performance of the Aptima ${ }^{\oplus}$ HV-1 Quant Dx Assay on the Panther System. Int J Med Health Biomed Bioeng Pharm Eng. 2015:9(5):397-400.

20. Nair SV, Kim HC, Fortunko J, Foote T, Peling T, Tran C, Nugent CT, Joo $S$, Kang Y, Wilkins B, Lednovich K, Worlock A. Aptima HIV-1 Quant Dx-A fully automated assay for both diagnosis and quantification of HIV-1. J Clin Virol. 2016;77:46-54

21. Sam SS, Kurpewski JR, Cu-Uvin S, Caliendo AM. Evaluation of performance characteristics of the Aptima HIV-1 Quant Dx Assay for the Detection and Quantitation of Human Immunodeficiency (HIV) Virus Type 1 in Plasma and CVL Samples. J Clin Microbiol. 2016;54:1036-41.

22. Buonaguro L, Tornesello ML, Buonaguro FM. Human Immunodeficiency Virus Type 1 Subtype Distribution in the Worldwide Epidemic: Pathogenetic and Therapeutic Implications. J Virol. 2007;81:10209-19.

23. Hopkins M, Hau S, Tiernan C, Papadimitropoulos A, Chawla A, Beloukas A, Gerretti AM. Comparative performance of the new Aptima HIV-1 Quant Dx assay with three commercial PCR-based HIV-1 RNA quantitation assays. Clin Virol. 2015;69:56-62.

24. Schalasta G, Borner A, Speicher A, Martin E. Comparative evaluation of the Aptima HIV-1 V2.0 assay using the Roche High Pure System for the quantification of HIV-1 RNA in plasma. Clin Chem Lab Med. 2016;54:493-9.

25. Mor O, Gozlan Y, Wax M, Mileguir F, Rakovsky A, Noy B, Mendelson E, Levy I. Evaluation of HIV-1RealTime, Xpert HIV-1 and Aptima HIV-1 Quant Dx asays in comparison to the Nuclisens EasyQ v2.0 assay for quantification of HIV-1 viral load. J Clin Microbiol. 2015;53(11):3458-65.

\section{Submit your next manuscript to BioMed Central and we will help you at every step:}

- We accept pre-submission inquiries

- Our selector tool helps you to find the most relevant journal

- We provide round the clock customer support

- Convenient online submission

- Thorough peer review

- Inclusion in PubMed and all major indexing services

- Maximum visibility for your research

Submit your manuscript at www.biomedcentral.com/submit

) Biomed Central 